\title{
Violencia intrafamiliar y abuso sexual: ¿Cómo integrarlos al círculo de pregrado de medicina?
}

\author{
Pamela Rojas González ${ }^{1}$, José Ramón Pinedo Palacios ${ }^{2}$
}

Resumen: Las cifras chilenas sobre abuso sexual (AS) y violencia al interior de las familias (VIF) son altísimas y transversales a todo estrato social. Un 25,9\% de los niños ha sido víctima de violencia grave en sus hogares (patadas, puñetazos, mordeduras, quemaduras, agresiones con armas) y un $8,7 \%$ refiere haber experimentado AS. Un tercio de las chilenas ha sufrido VIF, cifra que se tradujo en 45 mujeres fallecidas el año 2015. Los médicos están en una posición única para identificar víctimas y romper el ciclo de violencia, sin embargo su formación al respecto es mínima. El Instituto de Medicina de Estados Unidos plantea que el medio más eficaz para mejorar la respuesta a VIF es la educación de los médicos, y ha llamado a que las facultades entreguen las competencias necesarias a sus estudiantes. Distintas Escuelas de Medicina de EEUU y Canadá ya lo están haciendo; en Chile esto aún no ocurre. Entre 2013 y 2014, mediante un Proyecto FONDEDOC revisamos la formación actual de los estudiantes de medicina en pesquisa y abordaje inicial de pacientes víctimas de VIFAS, y generamos una propuesta para fortalecer el desarrollo de competencias afines en el currículo de pregrado. Este artículo presenta los productos finales de ese proyecto. Se discute sobre la discordancia entre la magnitud del problema en Chile y la formación actual de nuestros médicos, se entrega un listado de competencias básicas para pesquisar y brindar una primera respuesta, y se ofrece una propuesta para fortalecer el currículo de pregrado en esta área.

Palabras clave: educación médica; violencia doméstica; violencia intrafamiliar; abuso sexual; currículo médico, ciencias médicas.

Abstract: The figures on sexual abuse and domestic violence are very high in Chile and they cut across the whole span of socioeconomic classes. $25.9 \%$ of children have suffered severe violence at home (kicks, punches, bites, burns, weapon agressions) and $8.7 \%$ refer having experimented sexual abuse. One third of Chilean women have suffered domestic violence, which translates in 45 women dying for this cause during 2015. Doctors are in a unique positoin to identify victims and to cut the violence cycle, however their training on this regard is scarce. The Institute of Medicine of the USA postulates the most effective way of improving the response to domestic violence is educating doctors, and has made a call to faculties to provide the competencies needed to their students. Several medical schools in USA and Canada are already doing so; in Chile this is not happening yet. Between 2013 and 2014 through a FONDEDOC project we reviewed the existing curriculum of Medical School in relation to identifying and to initially approach patients suffering domestic violence-sexual abuse, and we generated a proposal to strengthen the development of pertinent competencies in undergraduate curriculum. This article exposes the product of this project. The discrepancy between the magnitude of the problem in Chile and the current training of our doctors is discussed. A list of basic competencies to identify and deliver the first response is presented, and a proposal to strengthen undergraduate curriculum in this area is offered.

Keywords: medical education; domestic violence; intimate partner violence; sexual abuse; medical curriculum; medical sciences.

Fecha de envío: 19 de mayo de 2016 - Fecha de aceptación: 30 de agosto de 2016

\section{Introducción}

El abuso sexual (AS) y la violencia al interior de las familias (VIF) son altamente prevalentes en Chile, y comprometen a todo estrato social. Esto sugiere que la probabilidad que uno de nuestros estudiantes se tope con un caso de VIF-AS es muy alta, sin embargo la mayoría de los estudiantes no integran la violencia como un diagnóstico diferencial. Esto hace que no la sospechen en sus pacientes, y por lo tanto, tampoco la diagnostiquen. Los médicos son cruciales en el proceso para identificar víctimas y romper el ciclo de violencia, sin embargo, sin un entrenamiento formal es fácil que un profesional minimice lo que observa o"no vea" lo que es evidente.

(1) Departamento Medicina Familiar. Escuela de Medicina Pontificia Universidad Católica de Chile.

(2) Departamento Psiquiatría. Escuela de Medicina Pontificia Universidad Católica de Chile.

Autor de correspondencia:projasgo@uc.cl 
El Instituto de Medicina de EEUU plantea que el medio más eficaz para mejorar la respuesta a VIF es la educación del médico. Ha recomendado que los conceptos relacionados a violencia doméstica y su denuncia sean aprendizajes prioritarios (Institute of Medicine, 2004), y ha hecho un llamado para que las facultades entreguen las competencias necesarias a sus estudiantes. Distintas entidades se han sumado a este llamado, y varias Escuelas de Medicina de EEUU y Canadá ya lo están haciendo (University of California, Los Angeles; Southern Illinois University; University of California, San Francisco, etc). En Chile, esto aún no ocurre.

Desarrollar las competencias necesarias para que los estudiantes de medicina puedan pesquisar y ofrecer una primera respuesta a pacientes víctimas de VIF-AS es un desafío, tanto por la necesidad de definir qué y cuánto debe aprender un estudiante, como también cómo debe hacerlo. Se ha propuesto, entre otras medidas, que las facultades entreguen las competencias centrales sobre VIF a sus estudiantes, y que los académicos asuman una mayor responsabilidad en desarrollar, probar y evaluar modelos o programas de formación innovadores al respecto (Institute of Medicine, 2002).

Los temas VIF-AS parecen ser "de nadie" en la formación médica chilena actual, pero de una u otra manera nos comprometen a todos. Nuestra propuesta es que la Escuela de Medicina de la Pontificia Universidad Católica y sus académicos, en función de la magnitud que tiene VIF-AS en Chile y la responsabilidad social que le es propia, se sumen a este llamado.

El objetivo de este artículo es difundir los productos y aprendizajes generados a partir de un proyecto de investigación docente, cuyo propósito fue generar una estrategia que permitiera al alumno de medicina UC adquirir las competencias para pesquisar y abordar inicialmente un caso de VIF-AS.

\section{Metodología}

Durante el período Agosto 2014 - Julio 2015 se condujo un proyecto FONDEDOC (Fondo de Desarrollo de la Docencia de la Universidad Católica de Chile), cuyo propósito fue generar una estrategia que permitiera al estudiante de medicina UC adquirir las competencias para pesquisar y abordar inicialmente un caso de VIF-AS, desde un aprendizaje formal (Rojas et al., 2014).

- Se trabajó en la revisión de las estadísticas sobre VIF-AS en Chile y de algunos modelos explicativos de las dinámicas de generación y mantención de estos problemas.
- Se contactó por vía correo electrónico a 15 Escuelas de Medicina chilenas, y se les preguntó por la formación que sus estudiantes de pregrado recibían sobre VIF-AS. El correo fue enviado en dos oportunidades. En paralelo se contactó a los jefes de los cursos de pregrado de la Escuela de Medicina UC, y se solicitó y revisó los programas de los cursos afines vigentes al año 2014.

En un tercer tiempo se recopiló y analizó literatura concerniente a educación médica e inclusión curricular de contenidos de VIFAS, utilizando experiencias publicadas dentro y fuera de Chile. Se utilizó como punto de partida artículos relevantes conocidos por los autores, y luego se realizó revisión de sus referencias, búsquedas adicionales por autor, y búsquedas no sistemáticas en Medline y otras bases de datos.

La información fue agrupada y sistematizada, y se utilizó para determinar la pertinencia de ofrecer una formación específica sobre VIF-AS a los estudiantes de Medicina, para definir qué competencias deberían adquirir en su programa de estudios, y para realizar recomendaciones con el fin de promover la inclusión de estos contenidos en los planes de estudio.

Con posterioridad se definió un listado de competencias específicas sobre VIF-AS para el egresado de medicina UC. Para esto el equipo a cargo trabajó integrando en una tabla las competencias sugeridas por la literatura con las metas de aprendizaje recientemente definidas para el perfil de egreso UC de pregrado. Esta tabla fue trabajada con 29 profesionales agrupados en 4 equipos consultores, los que fueron seleccionados por conveniencia a partir de su experticia técnica y/o por su exposición laboral o académica a VIF-AS. La tabla 1 muestra como quedó integrado el equipo consultor.

Utilizando una metodología de consenso no estructurado, los 4 equipos consultores trabajaron guiados por la pregunta: "en función de las metas de aprendizaje establecidas por la Escuela de Medicina, ¿cuál de las siguientes competencias le parecen adecuadas para un egresado de la Escuela de Medicina UC?".

\section{Resultados}

Complejidad del tema VIF-AS: La revisión bibliográfica realizada da cuenta que VIF-AS corresponde a un fenómeno complejo, tanto por su magnitud, como por lo que ocurre con los profesionales de la salud que se enfrentan a estas situaciones sin una formación adecuada. 
Tabla 1: Grupos consultores para definición de competencias deseables para el egresado de Medicina UC en VIF-AS

\begin{tabular}{|c|c|c|}
\hline Grupo & Integrantes & $\mathbf{N}$ \\
\hline $\begin{array}{l}\text { Atención } \\
\text { Primaria }\end{array}$ & $\begin{array}{l}\text { Equipo Salud Mental CESFAM Ancora San } \\
\text { Alberto Hurtado ( } 4 \text { Psicólogos, } 3 \text { médicos, } 2 \\
\text { trabajadoras sociales) } \\
\text { Equipo Supervisores clínicos Internado } \\
\text { Medicina Familiar CESFAM Ancora San } \\
\text { Alberto Hurtado (5) }\end{array}$ & 16 \\
\hline $\begin{array}{l}\text { Académicos } \\
\text { Pediatría UC }\end{array}$ & $\begin{array}{l}\text { Jefe Curso Pediatría } 5^{\circ} \text { año, Jefe Internado } \\
\text { Pediatría }\end{array}$ & 2 \\
\hline $\begin{array}{l}\text { Académicos } \\
\text { Psiquiatría UC }\end{array}$ & $\begin{array}{l}\text { Incluye Psiquiatra infantil y Psiquiatra } \\
\text { Adultos }\end{array}$ & 2 \\
\hline $\begin{array}{l}\text { Académicos } \\
\text { Medicina } \\
\text { Familiar UC }\end{array}$ & $\begin{array}{l}\text { Incluye médicos familiares mención adulto } \\
\text { (3), mención niño (5), y Jefe Internado } \\
\text { Medicina Familiar }\end{array}$ & 9 \\
\hline
\end{tabular}

El AS y VIF son problemas de salud altamente prevalentes en Chile. El 71\% de nuestros niños sufre VIF; 25,9\% experimenta violencia grave (patadas, puñetazos, golpizas, mordeduras, agresiones con armas, quemaduras); y el 8,7\% declara haber experimentado AS (Larraín et al., 2012). Por su parte, cerca de un 30\% de las chilenas refiere haber sufrido VIF (SERNAM, 2013), situación que el 2015 significó la muerte de 45 mujeres (SERNAM, 2015). Cuando estas prevalencias se estiman en población consultante a servicios de salud las cifras se elevan. Una revisión sistemática chilena publicada el año 2014 mostró prevalencias de vida de VIF entre un 50 y 80\%, cifras que los investigadores explican por la relación potencial entre VIF y salud (León et al., 2014). Es importante recordar que nuestros estudiantes toman contacto precisamente con este grupo de personas, o sea, población consultante a servicios de salud.

Para el decenio actual, Chile se ha propuesto como objetivo sanitario reducir la morbilidad, discapacidad y mortalidad prematura asociadas a violencia, trazando como meta sanitaria "disminuir la tasa de femicidios en mayores de 15 años"(MINSAL, 2011). Las prevalencias presentadas y la meta que se ha trazado el país convierten a VIF-AS en temas prioritarios para la salud de Chile.

Distintos autores concuerdan con que minimizar, e incluso negar lo ocurrido, es parte de las estrategias que usan tanto las víctimas como sus familiares para proteger su equilibrio frente a situaciones tremendamente complejas, dolorosas y difíciles de explicar. Los profesionales de la salud que los atienden, y que son testigos de sus relatos y de los signos clínicos de la violencia, se ven afectados por el mismo fenómeno. Ninguno de los actores involucrados logra "ver" ni "nombrar" fácilmente estas situaciones, aun cuando puedan ser evidentes (Barudy, 1998; Ravazzola, 1997).
La literatura internacional propone que en el terreno de las relaciones violentas hay muchos trucos invisibilizadores en los que un profesional sin un entrenamiento formal puede caer fácilmente. Por una parte están los agresores, que logran justificar sus prácticas con argumentos sólidos y confundentes. Por el otro lado, hay víctimas que se visualizan a sí mismas en una posición jerárquica inferior, y que encuentran argumentos para justificar el actuar del agresor, e incluso apoyar sus conductas. Y por último, un tratante no entrenado en violencia, que aun frente a la evidencia, busca retornar a su equilibrio "no registrando" o "minimizando" lo que observa y asumiendo que la víctima posee más poder del que en realidad tiene (Barudy, 1998; Ravazzola, 1997).

¿Cuánta formación recibe un médico en relación a VIF-AS? No identificamos en la literatura nacional ningún reporte publicado acerca de este punto. En cuanto a la consulta realizada, sólo 3 de las 15 Escuelas de Medicina contactadas respondieron, 1 de Santiago y 2 de provincia, y describieron una experiencia bastante similar entre ellas; inclusión de contenidos de manera aislada sobre VIF-AS en algunos cursos entre $4^{\circ}$ y $6^{\circ}$ año (Medicina Legal, Ginecoobstetricia, Psicopatología), la mayoría de las veces tratados sólo de manera teórico expositiva. Sólo una universidad declaró retomar estos conceptos en forma posterior, durante su Internado de Salud Familiar, a través de un seminario único.

En la UC las instancias en las cuales nuestros estudiantes de medicina se forman en VIF-AS han aumentado en los últimos años. Sin embargo, su perfil es similar al reportado por las otras universidades y por la literatura. Sólo los cursos de Psiquiatría y Pediatría ( $5^{\circ}$ año), y el curso de Medicina legal $\left(7^{\circ}\right)$ incluyen contenidos específicos sobre "maltrato infantil y AS", los que se trabajan en sesiones expositivas únicas de 60 y 45 minutos respectivamente. La asistencia a estas actividades no es obligatoria, y no existe mayor articulación entre un curso y otro. Los internados de Pediatría, Psiquiatría y Medicina Familiar, por su parte, declaran como contenido de sus programas algunos tópicos sobre VIF-AS, sin embargo no los trabajan en actividades formales, de modo que su revisión queda a criterio del estudiante.

A nivel nacional no existe información sistematizada en relación a cuánta formación reciben los médicos en su pre y postgrado en relación a VIF-AS. Hay acuerdo en la literatura internacional, sin embargo, en que esta formación es escasa, con falta de contenidos en los currículos, y con un abordaje aislado y más bien teórico (Gerber et al., 2009; Hill, 2005; Jamner et al., 2000; Miranda et al. 2009; Narayan et al., 2006; Reece et al., 2005). 
Rojas et al.

Tabla 2: Competencias deseables para el médico egresado UC en relación a VIF-AS

\begin{tabular}{|c|c|}
\hline Dim & Competencia \\
\hline Cognitivo & $\begin{array}{l}\text { - Definir VIF-AS según la legislación chilena vigente. } \\
\text { - Conocer la epidemiología de VIF-AS en Chile. } \\
\text { - Conocer algunos modelos comprensivos de VIF-AS, factores de riesgo y protección; y sus rangos de expresión. } \\
\text { - Entender las consecuencias físicas, conductuales, psicológicas y emocionales de VIF-AS } \\
\text { - Conocer historias, conductas, actitudes, síntomas y signos sugerentes de VIF-AS, tanto en las víctimas como en sus acompañantes. } \\
\text { - Identificar preguntas apropiadas con las que los médicos pueden alentar a los pacientes a hablar sobre VIF-AS } \\
\text { - Comprender que los factores culturales, incluyendo etnia y ruralidad, influyen en las respuestas que la comunidad tiene frente a VIF-AS. } \\
\text { - Comprender por qué una víctima puede permanecer en una relación abusiva y las barreras para pedir ayuda } \\
\text { - Identificar mitos sobre VIF-AS. } \\
\text { - Conocer conceptos generales de intervención en crisis de primer orden } \\
\text { - Conocer los principales elementos para evaluar riesgo inmediato y planificar estrategias de seguridad para la víctima. } \\
\text { - Conocer el concepto de revictimización y sus consecuencias } \\
\text { - Conocer los requerimientos legales chilenos en relación a confidencialidad y denuncia. } \\
\text { - Conocer el procedimiento de denuncia y sus implicancias para el médico y la víctima (legales, reparación), e identificar mitos en } \\
\text { relación a éste. } \\
\text { - Identificar los recursos disponibles para las víctimas (equipos, comunidad, judicial, e institucionales), y su rol. } \\
\text { - Conocer los principios éticos en juego en relación a VIF-AS }\end{array}$ \\
\hline & $\begin{array}{l}\text { - Educar en prevención de VIF-AS desde su espacio clínico (supervisiones de salud, atenciones clínicas, etc.) } \\
\text { - Reconocer historias, conductas, actitudes, síntomas y signos sugerentes de VIF-AS, tanto en las víctimas como en sus acompañantes. } \\
\text { - Evaluar a los pacientes en los que se sospecha VIF- AS a través de una historia clínica y examen de salud adecuado (de manera } \\
\text { sensible, empática, acorde a su nivel de formación y evitando revictimizar) } \\
\text { - Comunicarse verbal y no verbalmente de manera apropiada frente a una situación de sospecha y/o revelación de VIF-AS } \\
\text { - Realizar una intervención en crisis de primer orden en víctimas de VIF-AS } \\
\text { - Expresar preocupación y apoyo, dentro de los límites profesionales apropiados. } \\
\text { - Documentar de manera rigurosa y no sesgada las declaraciones y observaciones clínicas realizadas. } \\
\text { - Evaluar el peligro y planificar la seguridad inmediata de la víctima } \\
\text { - Revisar junto al paciente los recursos comunitarios y legales disponibles. } \\
\text { - Derivar y asegurarse que los pacientes estén conectados con los especialistas y recursos necesarios. } \\
\text { - Negociar roles y responsabilidades con el equipo de salud para identificar, intervenir y seguir a estos pacientes. } \\
\text { - Educar a pacientes y sus familias acerca de los límites de la confidencialidad y los requerimientos de denuncia. } \\
\text { - Acatar la normativa legal chilena en relación a confidencialidad y denuncia. }\end{array}$ \\
\hline Act & $\begin{array}{l}\text { - Reconocer que el cuidado médico entrega una oportunidad de interrumpir el ciclo de violencia en individuos, familias y } \\
\text { comunidades, demostrando una actitud de gravedad y urgencia sobre ésta como problema de salud } \\
\text { - Asumir como responsabilidad profesional la identificación de víctimas, y proveer una intervención adecuada. } \\
\text { - Reconocer que los valores, actitudes, creencias y experiencias personales que los profesionales tengan o hayan tenido en VIF-AS } \\
\text { puede afectar el que ellos sospechen, indaguen, e intervengan en casos de este tipo. } \\
\text { - Aceptar las respuestas de los involucrados en VIF-AS de manera comprensiva y sin emitir juicios de valor, aceptando que la } \\
\text { víctima pueda no reconocerse como tal. } \\
\text { - Reconocer y respetar el derecho de cada paciente mayor de edad a la autodeterminación, reconociendo que su } \\
\text { empoderamiento puede ser un proceso de largo plazo. } \\
\text { - Reconocerse como potencial agente revictimizante } \\
\text { - Entenderse como miembro de un equipo de salud, valorando la contribución de otras profesiones en la prevención y manejo de } \\
\text { situaciones de VIF-AS. } \\
\text { - Asumir la responsabilidad de autoformación en VIF-AS según las exigencias de su cargo. }\end{array}$ \\
\hline
\end{tabular}


¿Qué formación debiera recibir un médico en VIF-AS? Las primeras aproximaciones para definir la formación que debería recibir un médico en violencia doméstica ocurrieron en marzo de 1994, en Oklahoma, a partir del trabajo de la Conferencia Nacional de Expertos en el Tratamiento de la Violencia Familiar. Esta fue la primera iniciativa que agrupó a profesionales de distintas disciplinas de la salud para desarrollar principios curriculares y objetivos de aprendizaje relacionados con VIF-AS. El trabajo de Oklahoma se continuó con el de la Asociación Americana de Escuelas de Medicina (AAMC, 1995) y luego con un número especial de la revista Academic Medicine (1997), relacionado con la integración de estos temas en la enseñanza médica (Brandt, 1997; Gerber et al., 2009; Hill, 2005; Jamner et al., 2000; Miranda et al. 2009; Reece et al., 2005).

Estas iniciativas pusieron de manifiesto la necesidad de asegurar la legitimidad de un currículo en evaluación de violencia familiar, como parte central de la educación de los profesionales de la salud (Brandt, 1997). Se plantearon distintos niveles de competencias en VIF-AS para los médicos, en función de la actividad que realicen, y un nivel básico de formación dirigido a todos los profesionales de la salud (Brandt, 1997; Gerber et al., 2009; Hill, 2005; Jamner et al., 2000; Miranda et al. 2009; Reece et al., 2005). Desde esa fecha distintos grupos han trabajado en definir las competencias que un médico debiera lograr en relación a VIF-AS.

Tres propuestas fueron seleccionadas por su profundidad y detalle de análisis: University of California Los Angeles (UCLA) (Jamner et al., 2000), Academy on Violence and Abuse (AVA) (Ambuel et al., 2011) y Alpert et al. (1997). Estas se integraron y trabajaron en conjunto con las metas de aprendizaje recientemente definidas para el egresado de medicina UC y se utilizaron como insumo para el trabajo realizado por los cuatro equipos consultores, bajo la metodología de consenso no estructurado. El resultado fue la definición de un listado de "Competencias deseables para el médico egresado UC en relación a VIF-AS", agrupadas por dominios, que se detalla en la tabla 2.

¿Cómo debiera integrarse al currículo la formación en VIF-AS? (Alpert et al., 1997; Brandt, 1997; Hamberger, 2007; Weiss et al., 2000) Si bien la literatura muestra que no hay consenso sobre cómo fortalecer los currículos de formación médica en relación a VIF-AS, la tendencia actual es hacerlo a través de programas longitudinales de aprendizaje que articulen competencias de distinto nivel. Una de las estrategias puestas en marcha por las Escuelas de Medicina Americanas ha sido promover el abordaje de estos temas en los contenidos de los cursos básicos y clínicos, a través de estrategias de integración curricular. Estos espacios de integración trabajan conceptos de distintas disciplinas (anatomía, fisiopatología, contenidos clínicos, tópicos sobre comunicación, aspectos culturales, legales, etc.). Los contenidos se trabajan a partir de casos clínicos integradores, en los cuales el foco está puesto en el paciente y su problema de salud, y donde convergen al mismo tiempo contenidos de distinta naturaleza y disciplinas de estudio.

La tabla 3 resume algunas recomendaciones para la integración curricular de competencias sobre VIF-AS.

\section{Discusión y conclusiones}

En Chile, pese a que el $25,9 \%$ de nuestros niños sufre violencia física grave por parte de quienes deben protegerlos, y que un $8,7 \%$ declara haber vivido situaciones de abuso sexual, sólo ofrecemos 3 sesiones expositivas a nuestros estudiantes relacionadas al tema. La discordancia es evidente y se repite con lo que ocurre en población adulta; al 31\% de las chilenas que refiere haber sufrido VIF, y a las 45 que mueren cada año a mano de sus parejas, no les ofrecemos más que la declaración sobre un "contenido" en los programas que estructuran la formación de nuestros médicos.

Los médicos y otros profesionales de la salud son cruciales en el proceso de prevención y diagnóstico de VIF-AS. Con frecuencia son los primeros en tomar contacto con las víctimas, y muchas veces los únicos depositarios de su confianza. Nuestra posición es única para identificar individuos y poblaciones en riesgo, y para implementar iniciativas de prevención e intervención.

Como escuela compartimos el desafío de otras universidades de enfrentarnos a condiciones adversas: tenemos estudiantes con un contacto clínico limitado en términos de tiempo; un gran número de campos clínicos que complejiza la tarea de estandarizar la docencia; y contamos con una diversidad de pacientes y estudiantes. Sumado a estos desafíos, la legislación que nos rige no nos obliga a formar a nuestros estudiantes en temas de VIF-AS, y el perfil de egresados y la prueba de acreditación que los mide en Chile, apenas considera estos temas como relevantes.

Para hacer frente a las distintas expresiones de violencia doméstica y al abuso sexual, Chile cuenta hoy con guías de salud que orientan la pesquisa, derivación y seguimiento de niños y adultos víctimas de estos problemas de salud (MINSAL, 1998; MINSAL, 2013; MINSAL-UNICEF, 2011; SERNAM, 2013). Existe una legislación específica al respecto, y una red, tanto a nivel sanitario como judicial que se ha montado y fortalecido para apoyar y reparar a quienes están siendo vulnerados a través de la violencia. No contamos, sin embargo, con una formación que permita a nuestros egresados sospechar, diagnosticar e intervenir de manera inicial a este grupo. 
Rojas et al.

Tabla 3. Recomendaciones específicas para integrar el tema de la violencia doméstica en el currículo de las disciplinas de la salud (Alpert et al., 1997; Brandt, 1997; Hamberger, 2007; Weiss et al., 2000)

\begin{tabular}{ll}
\hline Recomendación & Justificación \\
\hline $\begin{array}{l}\text { La formación en aspectos } \\
\text { de VIFy AS no debe ser } \\
\text { exclusivamente autodirigida. }\end{array}$ & Se recomienda una formación dirigida y guiada, con actividades formales, integradas a un programa de estudios. \\
\hline $\begin{array}{l}\text { Aprovechar oportunidades } \\
\text { existentes (Hamberger, } \\
\text { 2007; Weiss et al., 2000) }\end{array}$ & $\begin{array}{l}\text { En especial las que ofrece el diseño curricular y los recursos académicos de la institución. Ej. Escuelas de medicina que } \\
\text { utilicen una metodología de enseñanza basada en problemas pueden revisar y modificar ciertos casos clínicos en uso } \\
\text { para introducl problema de la violencia interpersonal. }\end{array}$ \\
\hline
\end{tabular}

Conseguir el apoyo de líderes y de los principales involucrados (Magrane et al., 2000; Weiss et al., 2000)

Identificar a facultativos expertos en el tema (Alpert et al., 1997; Hamberger, 2007; Weiss et al., 2000)

Incorporar un equipo multidisciplinario
Para introducir cambios en el currículo es esencial contar con el apoyo de directores, comités, administradores y estudiantes, quienes deben reconocer la importancia que tiene la educación en este tema, utilizando así su influencia y habilidad en el logro de los cambios necesarios.

\section{Contar, en las distintas facultades y en la comunidad con facultativos competentes e interesados, permite entrenar y supervisar a los estudiantes en las competencias relevantes.}

Se sugiere que en el desarrollo e implementación de un currículo sobre violencia trabaje un equipo integrado por miembros de distintas disciplinas y especialidades, quienes sientan como propia la necesidad de incorporar el tema en sus ámbitos de trabajo y en la educación de los estudiantes (Alpert et al., 1997; Brandt, 1997; Magrane et al., 2000; Weiss et al., 2000).

Como explican Alpert et al. (1997), especialidades como Pediatría y Medicina de Urgencia pueden abordar los aspectos clínicos de este problema, mientras que disciplinas como Ética, Salud Pública y Medicina Legal entregarán una perspectiva social.

Planificar un currículo de esta manera permite a su vez entrenar a los estudiantes dentro del enfoque de trabajo en equipo que requiere el abordaje de este problema (Weiss et al., 2000).

Analizar la cultura de la institución (Alpert et al., 1997)

Facilitar y desarrollar recursos educativos (Alpert et al., 1997)
De modo que los objetivos y metas curriculares propuestas sean coherentes con las de la institución y se facilite así su integración.
Se propone contar con una plataforma con información actualizada y relevante que facilite a los académicos los recursos educativos necesarios. Se recomienda, incluso, aprovechar esta instancia para formar centros de investigación, tratamiento y prevención, que pueden apoyar el currículo y posicionar a la escuela como centro experto en el abordaje de este problema.

Se recomienda que las organizaciones profesionales y de acreditación incorporen el problema de la violencia interpersonal en sus propuestas de currículo y principios guías, y también en los exámenes de acreditación. Esto facilitaría que las escuelas integren el tema en sus objetivos educativos

acreditación (Alpert et al. 1997; Brandt, 1997)

Persistir (Brandt, 1997; Magrane et al., 2000; Weiss et al., 2000)

La introducción del tema de violencia interpersonal en la educación médica puede enfrentar múltiples resistencias de parte de miembros de la facultad y estudiantes, a raíz de la competencia por tiempo en el currículo o por la incomodidad que pueden experimentar al tratar este asunto. Su introducción e integración puede requerir tiempo, esfuerzo y múltiples revisiones.

Dada la magnitud de VIF-AS en Chile, y la dificultad de enfrentar estos temas sin la formación adecuada, ponemos en relevancia la necesidad de incluir de manera explícita contenidos afines en el programa de estudios del estudiante de medicina. Este gesto apunta a legitimar VIF-AS como problemas de salud relevantes en nuestro país, pero por sobre todo a convertir un "tema de nadie" en una meta "de todos" quienes ejercemos la medicina. Ajustar los currículos a las necesidades del país y de los futuros profesionales es una responsabilidad ineludible para una Universidad, y esta publicación apunta específicamente a hacer visible esta propuesta.
Contribuciones y reconocimientos: En esta sección se detallan las contribuciones de cada autor al trabajo, las fuentes de financiamiento y los posibles conflictos de interés declarados por los autores. Srta. Claudia Parra. Licenciada en psicología, Pontificia Universidad Católica de Chile. Por su trabajo bibliográfico durante el desarrollo del proyecto. Financiamiento: Este proyecto fue desarrollado al interior del Departamento de Medicina Familiar de la Pontificia Universidad Católica de Chile, bajo el financiamiento del Fondo de Desarrollo de la Docencia (FONDEDOC) 2014 de dicha institución. 
Rojas et al.

\section{Referencias}

AlpertEJ, Sege RD \& BradshawYS (1997). Interpersonal violence and the education of physicians. Academic Medicine 72 (Supplement), S41-50.

Ambuel B, Trent K, Lenahan P, Cronholm P, Downing D, Jelley $M$, Lewis-O'Connor A, McGraw M, Marjavi A, Mouden L, Wherry J, Callahan M, Humphreys J \& Block R (2011). Competencies Needed by Health Professionals for Addressing Exposure to Violence and Abuse in Patient Care. Academy on Violence and Abuse. Disponible en http://www.nsvrc.org/sites/default/files/CoreCompetenciesRevApril2011.pdf Accedido el 16 de mayo, 2016.

Barudy J (1998). El dolor invisible de la infancia. Una lectura ecosistémica del maltrato infantil. Paidós Ibérica, Barcelona.

Brandt EN Jr. (1997). Curricular principles for health professionals'education about family violence. Academic Medicine 72 (Supplement), S51-58.

Gerber MR \& Tan AK (2009). Lifetime intimate partner violence exposure, attitudes and comfort among Canadian health professions students. BMC Research Notes 2, 191.

Hamberger LK (2007). Preparing the next generation of physicians: medical school and residency-based intimate partner violence curriculum and evaluation. Trauma Violence Abuse 8(2), 214-225.

Hill JR (2005). Teaching about family violence: a proposed model curriculum. Teach Learn Med 17(2), 169-178.

Institute of Medicine (US). Committee on the Training Needs of Health Professionals to Respond to Family Violence, Board on Children, Youth, and Families. Cohn F, Salmon ME, Stobo JD, Editors. (2002). Confronting Chronic Neglect: The Education and Training of Health Professionals on Family Violence. National Academies Press (US), Washington, D.C.

Institute of Medicine (US). Committee on Behavioral and Social Sciences in Medical School Curricula. Cuff PA, Vanselow NA, Editors. (2004). Improving Medical Education: Enhancing the Behavioral and Social Science Content of Medical School Curricula. National Academies Press (US), Washington, D. C. Disponible en http://www. ncbi.nlm.nih.gov/books/NBK10238/Accedido el 09 de febrero, 2015.

Jamner MS \& Stokols D, Editors. (2000). Promoting Human Wellness: New Frontiers for Research, Practice, and Policy. University of California Press, Berkeley. Disponible en http://ark.cdlib.org/ ark:/13030/kt4r29q2tg/ Accedido el 04 febrero, 2015.

Larraín S \& Bascuñán C (2012). Cuarto Estudio de Maltrato Infantil UNICEF. Santiago, Chile. Disponible en http://unicef.cl/web/cuarto-estudio-de-maltrato-infantil/ Accedido el 17 de mayo, 2016.
León T, Grez M, Prato J, Torres R \& Ruiz S (2014). Violencia intrafamiliar en Chile y su impacto en la salud: una revisión sistemática. Revista Médica de Chile 142(8), 1014-1022.

Magrane D, Ephgrave K, Jacobs M \& Rusch R (2000). Weaving women's health across clinical clerkships. Academic Medicine 75, 1066-1070.

MINSAL (1998). Manual de apoyo técnico para las acciones de salud en violencia intrafamiliar. Pp. 23-31. Disponible en http://web. minsal.cl/portal/url/item/71e55238a2042745e04001011f01638a. pdf Accedido el 10 de febrero, 2015.

MINSAL (2011). Estrategia Nacional de Salud Para el Cumplimiento de los Objetivos Sanitarios de la Década 2011-2020. Disponible en http://www.minsal.cl/portal/url/item/c4034eddbc96ca6de0400101640159b8.pdf Accedido el 24 de octubre, 2016.

MINSAL (2013). Guía Clínica: Detección y primera respuesta a niños, niñas y adolescentes víctimas de maltrato por parte de familiares o cuidadores. Disponible en http://web.minsal.cl/sites/default/files/files/ Guia_maltrato_Valente26dic2013.pdf Accedido el 10 de febrero, 2015.

MINSAL-UNICEF (2011). Atención de Niños, Niñas y Adolescentes Menores de 15 Años, víctimas de abuso sexual. Disponible en http:// web.minsal.cl/portal/url/item/aaa27720f363a745e04001011e011120. pdf Accedido el 10 de febrero, 2015.

Miranda E, Garmendia F, Perales A, Mendoza P, Miano J \& Calderón W (2009). Evaluación de los planes curriculares y sílabos de las asignaturas de la Facultad de Medicina de la UNMSM, sobre la atención integral en salud a personas afectadas por la violencia. An Fac Med 70(4), 273-276.

Narayan AP, Socolar RR \& St Claire K (2006). Pediatric residency training in child abuse and neglect in the United States. Pediatrics 117(6), 2215-2221.

Ravazzola M (1997). Historias infames: los malos tratos en las relaciones. Paidós Ibérica, Buenos Aires.

Reece RM \& Jenny C (2005). Medical training in child maltreatment. Am J Prev Med 29(5 Suppl 2), 266-271.

Rojas P \& Pinedo J (2014). Estrategias innovadoras para mejorar la pesquisa y abordaje inicial de casos de abuso sexual y violencia intrafamiliar. FONDEDOC 2014.

SERNAM (2013). Encuesta de Victimización por VIF y Delitos Sexuales. Disponible en https://estudios.sernam.cl/documentos/?eMjM1MTAxOQ==-Encuesta_Nacional_de_Victimizaci\%C3\%B3n_por_Violencia_Intrafamiliar_y_Delitos_Sexuales_2012 Accedido el 17 de mayo, 2016. 
Rojas et al.

SERNAM (2013). Plan nacional de violencia intrafamiliar en Chile. Disponible en http://www.sernam.cl/descargas/Plan_Nacional_2012-2013.pdf. Accedido el 10 de febrero, 2015.

SERNAM (2015). Femicidios 2015. Disponible en https://portal. sernam.cl/img/upoloads/FEMICIDIOS\%202015\%20actualizado\%20 al\%2028\%20de\%20diciembre.pdf Accedido el 14 de mayo, 2016.
Weiss LB, Coons HL, Kripke EN \& O'Brien MK (2000). Integrating a domestic violence program into a medical school curriculum: Challenges and strategies. Teaching and Learning in Medicine 12, 133-140. 